\title{
Los costes de un programa de cirugía menor durante un año en un centro de Atención Primaria de Salud
}

J. Cruzado Quevedo, J. Hernández Soler, P. Alcántara Muñoz, F.J. López Román,

C. Contreras García, C. SÁnchez Quiles ${ }^{1}$ Médicos de Familia. 'Residente de tercer año de MFyC. Centro de Salud Virgen de la Consolación. Molina de Segura. Unidad Docente de Medicina Familiar y Comunitaria. GAP Número 1.

Servicio Murciano de Salud. Murcia

\section{RESUMEN}

Fundamentos: existen varios estudios sobre experiencias y efectividad de programas de cirugía menor (CM) en Atención Primaria (AP) pero hay mucho desconocimiento acerca de los costes de estas actividades para poder establecer comparaciones entre los diferentes niveles asistenciales y áreas geográficas.

Objetivo: medir los costes de un año de práctica de CM en un centro de AP.

Diseño: estudio de evaluación económica, descriptivo, retrospectivo.

Material y métodos: se desarrolló en AP sobre 479 pacientes tratados con diferentes procedimientos de CM desde enero a diciembre de 1998. Se incluyeron pacientes con patologías dérmicas y de uña que recibieron procedimientos quirúrgicos programados. Se excluyeron aquéllos con lesiones dérmicas que necesitaron tratamiento inmediato, sospecha de lesión maligna, queloides previos, riesgo de lesión neurológica, alergia a anestésicos locales, tratamiento anticoagulante.

Medimos por separado costes de crioterapia y costes de otros procedimientos de CM.

Resultados: fueron evaluadas 336 sesiones de criocirugía en 267 pacientes con un coste total anual de 589.857 ptas. $(3.545,11 €)$. El coste medio por procedimiento fue 1.755 ptas. $(10,55 €)$. El coste medio por paciente fue 2.209 ptas. $(13,28 €)$.

También fueron evaluadas 212 sesiones de otros procedimientos de CM en 212 pacientes con un coste total anual de 1.627.228 ptas. (9.779,8€). El coste medio por paciente y procedimiento fue 7.676 ptas. $(46,13 €)$.
The costs of a program of minor surgery during one year in a Primary Health Care centre

\section{ABSTRACT}

Backgrounds: there are some studies about experience and effectiveness of minor surgical procedures (MS) in Primary Health Care (PHC) but there are still many unknowns about the cost of these activities to be able comparisons of their results among different levels of care and geographical areas.

Objective: to measure the costs of MS practice for a year in PHC centre.

Design: retrospective descriptive cost analysis study.

Material and methods: this study was developed in PHC over 479 patients treated with different MS procedures since January until December of 1998. We included all patients suffered from skin lesions or toenails pa-thologies who were treated with programmed surgeries procedures. We excluded patients suffered from skin lesions and required an immediate treatment, suspected to have a malignant lesion, or some previous keloides, being under risk of presenting neurological injury, being allergic to local analgesic, or being under anticoagulation treatment.

The costs of cryotherapy and the costs of other MS procedures were evaluated separately.

Results: we evaluated a total of 336 cryotherapy sessions in 267 patients. The annual total cost was 589,857 ptas. (3,545.11€). The average cost per procedures was 1,755 ptas. (10.55€). The average cost per patient was 2,209 ptas. (13.28€).

Also, we evaluated a total of 212 sessions of other minor surgery procedures in 212 patients. The annual total cost was 1.627.228 ptas. (9.979€). 
Conclusiones: la falta de estudios de costes sobre CM en especializada en nuestra región impide comparar nuestros resultados. Aunque en nuestra opinión, los programas de CM en AP muestran un bajo coste, se precisa utilizar metodologías estandarizadas de evaluación económica para poder comparar resultados tanto en AP como especializada.

Palabras clave: Cirugía menor. Atención Primaria. Evaluación económica.
The average cost per patient was 7,676 ptas (46.13€).

Conclusion: we have not found any similar study in Secondary Health Care (SHC) in our region to compare with our study. In our opinion, the minor surgery programs in PHC show a low cost. It is necessary to use standardized methods of economic evaluation in PHC and SHC to compare results.

Key words: Minor surgery. Primary Health Care. Cost analysis.

\section{INTRODUCCIÓN}

Desde hace algunos años se vienen realizando programas de cirugía menor $(\mathrm{CM})$ en Atención Primaria (AP) en España, entendiendo como CM aquellos procedimientos que tienen como característica común la aplicación de procedimientos quirúrgicos u otras intervenciones sencillas y de corta duración, que se realizan habitualmente en los tejidos superficiales y/o accesibles y que precisando de anestesia local tienen poco riesgo y complicaciones escasas ${ }^{1}$. Aunque hay estudios en nuestro medio sobre experiencias y efectividad de dichos programas $^{2-4}$, poco se sabe de los costes que supone dicha actividad. Tan sólo dos artículos de reciente publicación analizan el impacto económico de la implantación de un programa de $\mathrm{CM}$ en $\mathrm{AP}^{5} \mathrm{y}$ valoran la eficiencia de la $\mathrm{CM}_{\text {en }} \mathrm{AP}^{6}$.

Otros artículos, realizados fuera de nuestro país, han analizado los distintos tipos de intervenciones de CM en AP y evaluado sus costes. Los resultados obtenidos son heterogéneos ya que existe controversia acerca de si su práctica es más económica cuando es realizada en AP o si es realizada en especializada. Así, un autor de práctica generalista, encuentra que la realización de CM en AP es 15 veces más barata que la misma actividad realizada en el ámbito hospitalario ${ }^{7,8}$. Otros autores, difieren del cálculo de estos costes y sugieren que para calcular los costes reales de cirugía menor se debería añadir los costes de otras actividades como son los estudios anatomopatológicos realizados'. Brown et al analizan las actividades de cirugía menor en Atención Primaria y encuentran que son costoefectivas ${ }^{10}$.

Por otro lado, resultados contrarios a la práctica de $\mathrm{CM}$ en AP son aportados por dermatólogos. Éstos sugieren que los costos de la práctica de CM pueden ser más baratos si los pacientes son vistos y tratados por ellos, ya que desde su punto de vista, numerosas intervenciones quirúrgicas sobre determinadas lesiones cutáneas, podrían ser obviadas o no recomendadas sobre la base de la mayor seguridad en el diagnóstico y la prescripción de un correcto tratamiento ${ }^{11,12}$.

El siguiente estudio, usando herramientas de evaluación económica ${ }^{13}$, intentó medir los costos de un año de práctica de cirugía menor en un centro de salud.

\section{MATERIAL Y MÉTODOS}

El diseño de estudio seleccionado para esta investigación es del tipo evaluación económica, descriptivo y retrospectivo. Fue desarrollado en el ámbito de AP tomando como población analizada a todos los pacientes mayores de 14 años de las dos zonas básicas de salud de Molina de Segura (Murcia) y en los que se realizó algún procedimiento de cirugía menor entre el 1 de enero de 1998 y el 31 de diciembre de 1998, quedando éste registrado en su historia clínica y en un registro específico nominal de intervenciones. Todos los pacientes de ambas zonas de salud tuvieron acceso al programa de $\mathrm{CM}$ independientemente de estar o no asignado a uno u otro médico. La identificación de casos y recogida de datos de los pacientes se basó en estos registros.

Los criterios de inclusión de pacientes al programa de CM fueron aquéllos que presentaban alguna de las siguientes patologías: nevus, queratosis solar, molluscum contagiosum, queratosis seborreica, quiste epidermoide, lipoma, verruga plantar, verruga vulgar, verruga seborreica, dermatofibroma, quiste mucoide, uña encarnada y otras patologías de las uñas, y en los que se realizaron alguno de los siguientes procedimientos quirúrgicos programados: curetaje, escisión de la lesión superficial, extirpación de lesión subcutánea, extirpación de cuerpo extraño, extirpación de uña encarnada, electro cauterización y criocirugía.

Los criterios de exclusión de pacientes del programa de CM fueron aquéllos que precisaron ciru- 
gía no programada urgente: heridas, laceraciones y abscesos de forma aguda, pues necesitaron tratamiento inmediato. Los sospechosos de padecer lesiones malignas, los que habían presentado cicatrices queloidales previas o riesgo de lesión neurológica, los alérgicos a anestésicos locales, los que estaban bajo tratamiento anticoagulante o los que padecían de serios problemas orgánicos, no se intervinieron en nuestro medio y por tanto también fueron excluidos.

En el desarrollo del programa de CM participaron 7 médicos de familia y 8 enfermeras/os de ambas zonas de salud. Todos habían recibido algún curso o taller formativo para la realización de CM.

Las intervenciones de $\mathrm{CM}$ se realizaron en una consulta específicamente habilitada para tal fin.

\section{Medición de costes}

Esta evaluación fue realizada desde la perspectiva del proveedor de servicios y trata de medir costes tangibles directos e indirectos sanitarios expresados en pesetas de 1998. Los costes indirectos tales como costos ocasionados por el tiempo gastado por el paciente en acudir y esperar en la consulta, o por el coste del tiempo en baja laboral, no fueron valorados en este trabajo. Los costes intangibles, tales como los ocasionados por la ansiedad, dolor físico, etc., tampoco fueron medidos en este trabajo por la dificultad de su medición. Por último en esta evaluación tampoco se planteó tasa de descuento de costes por no beneficio ya que los datos recogidos fueron de un año ${ }^{13-16}$.

Para una mejor comprensión se subclasificaron los costes en costes derivados de la práctica de criocirugía y en costes de otros procedimientos de cirugía menor (curetaje, escisión de la lesión superficial, extirpación de lesión subcutánea, extirpación de cuerpo extraño, extirpación de uña encarnada, electro cauterización). Estos tratamientos se evaluaron por separado debido a las importantes diferencias de técnica y procedimiento entre ambos.

En este estudio se ha determinado el coste total anual de tratamiento por criocirugía y el coste total anual por otros procedimientos de cirugía menor. También se ha calculado el coste medio por paciente y lesión tratada durante el año 1998.

\section{Costes derivados de la práctica de criocirugía}

Fueron calculados como: a) costes previos a criocirugía que incluyen el coste del tiempo utilizado (6 minutos por consulta y paciente) en la primera visita al médico de familia; b) costes de los recursos humanos empleados en la práctica de criocirugía medidos como el coste de la suma del tiempo utilizado para cada paciente por médico de familia y/o enfermera/o en cada acto de criocirugía más el tiempo utilizado en los controles de revisión, incluyendo las revisiones de complicaciones; c) costes de material fungible utilizado medidos como los costes del nitrógeno líquido facturado en el año 1998 más el material usado en las complicaciones; d) costes administrativos calculados como la parte proporcional del total de los costes anuales de personal administrativo del CS por hora de utilización de la consulta para criocirugía; y e) gastos generales (luz, agua, teléfono, limpieza, lavandería, mantenimiento) calculados también como la parte proporcional del total de gastos generales anuales por hora de utilización de criocirugía.

Para cada uno de los pacientes candidatos a criocirugía se recogió, en una hoja de registro, cada actividad realizada con sus correspondientes tiempos, revisiones, complicaciones, etc. (Fig. 1). Si el proceso recidivaba después de la curación se consideraba como un nuevo caso y se registraba como tal.

\section{Costes de otros procedimientos de cirugía menor}

Incluyen todos los costes de otros procedimientos de cirugía menor y que son analizados como: a) costes previos al procedimiento empleado valorados de forma similar a como se describió en el apartado de criocirugía; b) costes de los recursos humanos empleados en el procedimiento de CM calculados como en el apartado de criocirugía; c) costes de material fungible utilizado en el procedimiento de $\mathrm{CM}$ y en las complicaciones: antisépticos, anestésicos locales, jeringuillas, agujas, hojas de bisturí, gasas, apósitos, suturas y sedas, esparadrapos, guantes, sabanitas, etc., y uso de antibióticos en el caso de infección; d) costes de esterilización del material usa$d o$ : valorados como el costo del tiempo utilizado por la auxiliar de enfermería más los materiales necesarios; e) costes de anatomía patológica (AP) que incluyen los costes de los análisis de las muestras enviadas a AP; f) costes administrativos; y g) gastos generales fueron calculados de forma similar al procedimiento de criocirugía.

También, para cada uno de los pacientes candidatos a otros procedimientos de cirugía menor, se recogió en una hoja de registro cada actividad realizada, tiempos, revisiones, remisión a AP, complicaciones, etc. (Fig. 2). Si el proceso recidivaba después de la curación se consideraba como un nuevo caso y se registraba de forma independiente.

\section{Valoración económica}

Para determinar el gasto mensual de material fungible, todos los meses se contabilizaba del al- 


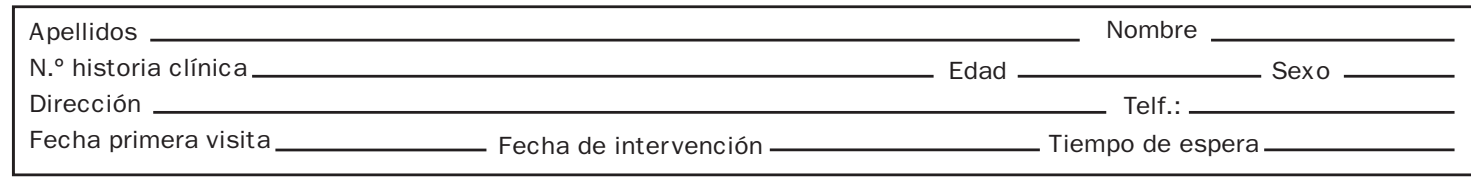

\begin{tabular}{|l|l|}
\hline $\mathbf{N}^{\mathbf{O}}$ & \multicolumn{1}{|c|}{ Diagnóstico clínico } \\
\hline & Nevus melanocítico \\
\hline & Queratoris actínica \\
\hline & Queratosis seborreica \\
\hline & Fibroma blando (acrocordón o f. péndulo) \\
\hline & Angioma \\
\hline & Verruga vulgar \\
\hline & Verruga plantar \\
\hline & Verruga plana \\
\hline & Verruga filiforme \\
\hline & Molusco contagioso \\
\hline & Otras patologías dermatológicas \\
\hline
\end{tabular}

Tiempo de descongelación de cada lesión (segundos):

Boquilla utilizada: __ A _ B _ _ _ _ D

Tiempo de duración de la intervención (desde el comienzo hasta el final) minutos

DIBUJO DE LAS LESIONES

Localización:

Tamaño:

N. ${ }^{\circ}$ de lesiones:

\begin{tabular}{|l|}
\hline \multicolumn{1}{|c|}{ Complicación: } \\
\hline Sin complicación \\
\hline Infección de la herida \\
\hline Pigmentación anómala de la cicatriz \\
\hline Disestesias \\
\hline Cicatriz antiestética \\
\hline Otras (especificar) \\
\hline
\end{tabular}

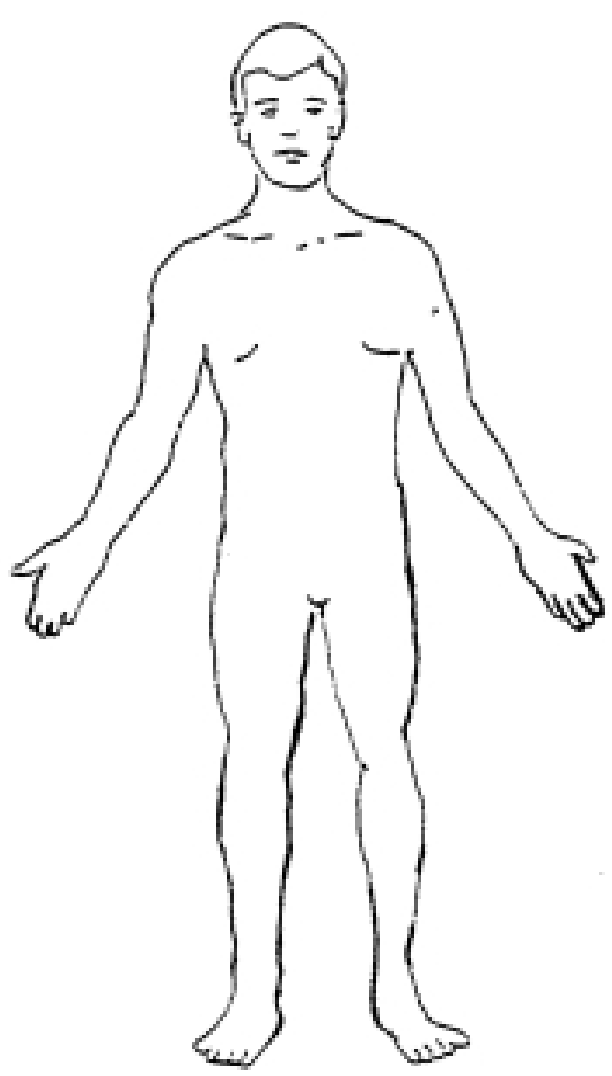

Resultado final de cada lesión:

\begin{tabular}{|c|l|l|l|}
\hline Lesiones & $\mathbf{1}^{\text {a }}$ congelación & $\mathbf{2}^{\text {a }}$ congelación & $\mathbf{3}^{\text {a }}$ congelación \\
\hline 1 & & & \\
\hline 2 & & & \\
\hline & & & \\
\hline & & & \\
\hline
\end{tabular}

Señalar con una C la sesión de congelación donde se cura la lesión y con una N si no cura

Fecha de alta: 


\begin{tabular}{lll} 
Apellidos & Nombre \\
N. ${ }^{\circ}$ historia clínica & Edad $\_$ & \\
Dirección & Sexo - Telf.: \\
Fecha primera visita & Fecha de intervención & Tiempo de espera \\
\hline
\end{tabular}

Médico

Enfermero/a

\begin{tabular}{|l|l|l|l|}
\hline Código & Diagnostico prequirúrgico & Tipo de intervención & Código \\
\hline & Nevus & Afeitado y cura & Excisión de lesiones superficiales \\
\hline & Queratosis solar & Extracción de lesiones & \\
\hline & Moluscum contagiosum & Punch & \\
\hline & Queratosis seborreica & Extracciones de cuerpos extraños \\
\hline & Quiste epidermoide & Extracción de uñas encarnadas & \\
\hline & Verrugas plantares & Electrocirugía & \\
\hline & Papiloma & & \\
\hline & Verrugas vulgares y seborreicas & & \\
\hline & Dermatofibroma & & \\
\hline & Quiste mucoide & & \\
\hline & Uña encarnada & & \\
\hline & Otras patologías de la uña & & \\
\hline & Otras lesiones cutáneas (especificar) & & \\
\hline
\end{tabular}

Localización de la lesión

Tamaño de la lesión

Tiempo de duración de la intervención (desde el comienzo hasta el final)

Se envía especimen para Anatomía Patológica

Sí No

\begin{tabular}{|l|l|l|l|}
\hline Código & Diagnostico histológico confirmado & Complicaciones & Código \\
\hline & Nevus & Sin complicaciones & Sangrado \\
\hline & Queratosis solar & Hematomas & \\
\hline & Moluscum contagiosum & Infección \\
\hline & Queratosis seborreica & Dehiscencia de puntos & \\
\hline & Quiste epidermoide & Secuelas & \\
\hline & Lipoma & Sin secuelas & \\
\hline & Verrugas plantares & Formación de queloides & \\
\hline & Verrugas vulgar y seborreica & Cicatriz dehiscente & \\
\hline & Dermatofibroma & Cicatriz pigmentada anormal & \\
\hline & Quiste mucoide & Cicatriz fea & \\
\hline & Otras lesiones cutáneas (especificar) & Déficit funcional & \\
\hline & Excisión Incompleta & Disestesesias \\
\hline & Sí & Otras (especificar) & \\
\hline & No & \\
\hline
\end{tabular}

Seguimiento y revisiones:

\begin{tabular}{|c|c|c|}
\hline & Médico & Enfermero/a \\
\hline & & \\
\hline & & \\
\hline & & \\
\hline
\end{tabular}

Fecha de curación

Figura 2

Hoja de registro de actividad para candidatos a otros procedimientos de cirugía menor. 
macén específico para cirugía menor los consumos de material y se anotaban en un estadillo/petitorio que se enviaba a la gerencia de AP para que ésta realizase la oportuna reposición. El gasto mensual de nitrógeno líquido fue facilitado por la empresa suministradora.

Los costes de los recursos humanos (médicos, enfermeras/os, auxiliar de clínica) fueron medidos en unidades de tiempo (horas de trabajo) y multiplicado por el precio de cada unidad. El coste de cada unidad de tiempo por grupo profesional se calculó sobre la parte proporcional del salario medio (precio de una hora por profesional). El salario medio se calculó como la suma de los salarios brutos de cada uno de los profesionales sanitarios que participaron en el programa (médicos por un lado, enfermeros/as por otros y auxiliar de clínica), más las cotizaciones sociales realizadas por la empresa dividido por el número de integrantes de cada sector y a su vez divididos por 1.645 horas que son el total de horas anuales por profesional. Los costes administrativos fueron calculados como el coste total de todo el personal administrativo del CS (salarios brutos más cotizaciones sociales) divididos por el número de consultas existentes en el centro y a su vez dividido por 1.645 horas de jornadas anuales. Para los costes generales se procedió de igual manera que los costes administrativos.

Toda la información de costes sobre personal sanitario y gastos generales fue facilitada por el departamento personal y de gasto de la gerencia de AP del área de salud $n^{\circ} 1$ de Murcia.

Los costes de materiales y de medicación fueron medidos en unidades y dosis y multiplicados por los precios actualizados facilitados por la gerencia de AP.

Los costes histopatológicos fueron facilitados por el Departamento de Anatomía Patológica del Hospital Morales Meseguer.

Todas las unidades fueron valoradas en precios actuales para el año 1998.

\section{RESULTADOS}

\section{Costes de criocirugía}

Durante el año 1998 se realizaron un total de 336 sesiones de criocirugía en 267 pacientes. Los costes previos a la criocirugía fueron de 160.430 ptas., los costes de los recursos humanos empleados en las sesiones de criocirugía y sus revisiones posteriores incluidas complicaciones ascendieron a 192.333 ptas., los costes materiales fueron de 204.439 ptas., los costes administrativos fueron de 25.149 ptas. y los gastos generales sumaron 7.506 ptas.
Por lo tanto; el coste total anual de la práctica de criocirugía fue de 589.857 ptas., el coste medio por lesión tratada con criocirugía fue de 1.755 ptas. y el coste medio por paciente fue 2.209 ptas. (Tabla I).

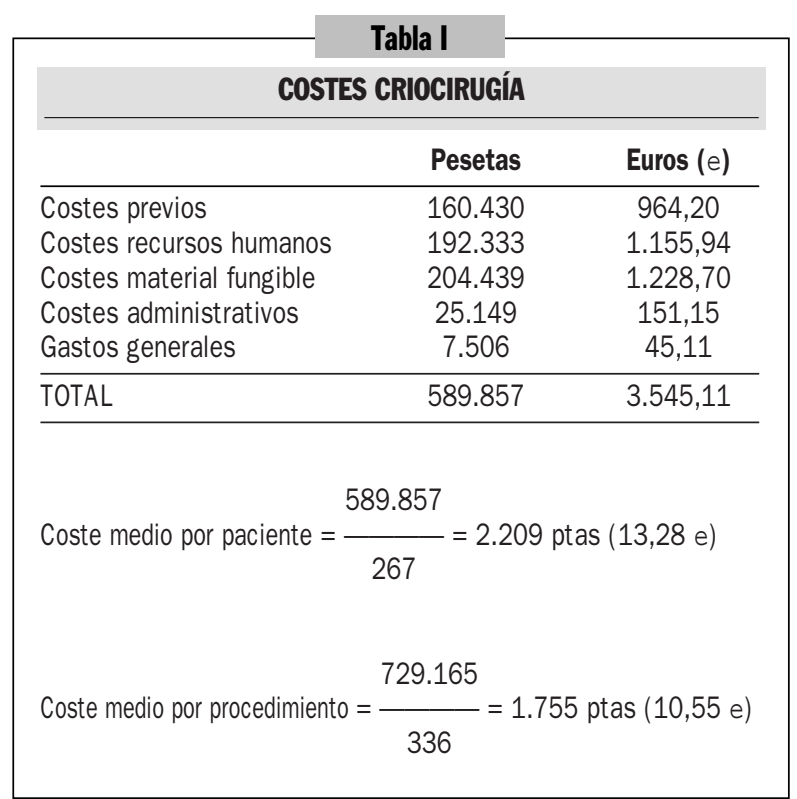

\section{Costes de otros procedimientos de cirugía menor}

Durante 1998 se realizaron un total de 212 procedimientos de cirugía menor (excluyendo criocirugía) en 212 pacientes. Los costes previos al procedimiento empleado fueron de 103.063 ptas., los costes de recursos humanos utilizados en los diferentes procedimientos de cirugía menor y sus revisiones posteriores incluidas complicaciones ascendieron a 686.711 ptas., los costes del material fungible fueron de 263.935 ptas., los costes de esterilización del material a usar fueron de 217.280 ptas., los costes de anatomía patológica por la remisión de 41 muestras a AP fueron de 264.632 ptas., los costes administrativos ascendieron 70.550 ptas. y los gastos generales sumaron 21.056 ptas.

Por tanto el coste total anual para los procedimientos de cirugía menor excluidos la criocirugía fue de 1.627 .228 ptas. y el coste medio por paciente y procedimiento cirugía menor excluida la criocirugía fue de 7.676 ptas. (Tabla II).

\section{DISCUSIÓN}

En los últimos años las evaluaciones económicas de las nuevas tecnologías sanitarias y de los nuevos medicamentos han conocido un auge importante. A la hora de realizar un estudio de evaluación económica se dispone de diversas metodologías. La falta de una metodología estandarizada supone una limi- 
Tabla II

\section{COSTES DE OTROS PROCEDIMIENTOS DE CIRUGIA MENOR}

\begin{tabular}{lrr}
\hline & Pesetas & Euros (e) \\
\hline Costes previos & 103.063 & 662,67 \\
Costes recursos humanos & 686.711 & $4.172,22$ \\
Costes material fungible & 263.935 & $1.586,28$ \\
Costes esterilización & 217.280 & $1.305,88$ \\
Costes de Anat. Patológ. & 264.632 & $1.590,47$ \\
Costes administrativos & 70.551 & 424,02 \\
Gastos generales & 21.056 & 126,55 \\
\hline TOTAL & 1.627 .228 & $9.779,84$ \\
\hline
\end{tabular}

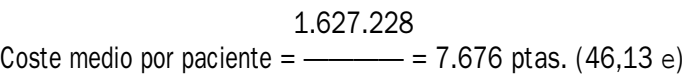

y procedimiento

212

tación importante por la variabilidad y la posibilidad de manipulación que supone ${ }^{17}$. En este trabajo, siguiendo a diferentes autores ${ }^{13-16}$, hemos intentado elaborar un método propio de medición de costes, por la necesidad de estandarizar para poder establecer comparaciones homogéneas en el futuro.

Nuestros resultados de 2.209 ptas. por paciente tratado con criocirugía, de 1.755 ptas. por lesión tratada con criocirugía y de 7.676 ptas. por paciente y procedimiento distinto al de criocirugía, sólo los hemos podido comparar con los resultados de un reciente estudio realizado por Tárraga López et $\mathrm{al}^{5}$, que encuentra un coste medio por intervención de $\mathrm{CM}$ en AP fue de 6.673 ptas. Las diferencias encontradas probablemente son debidas a las diferentes formas de calcular los costos y a que en nuestro estudio se incluyen los costos administrativos y gastos generales. En relación al estudio recientemente publicado por Vaquero Martínez et $\mathrm{al}^{6}$ que encuentra un coste de 1.431 ptas. por procedimiento. Estas diferencias se pueden explicar debido a que estos autores no tienen en cuenta los costes de personal, ni los gastos administrativos, ni de mantenimiento, ni tampoco los gastos de los estudios anatomopatológicos. Por último este estudio no separa los costos de los procedimientos de criocirugía de los costes de otros procedimientos de CM, siendo los primeros mucho más económicos, tal y como queda evidenciado en nuestro estudio. Por ello insistimos en que sería interesante establecer una metodología estandarizada de valoración de costes.

Por otro lado no nos parece útil comparar nuestros resultados, obtenidos en el ámbito de la sanidad pública, con los costes de las intervenciones de procedimientos de CM en el ámbito de la sanidad privada, por que obviamente estos últimos conllevan implícitamente añadidos unos beneficios empresariales.

No hemos podido comparar los costes de similares procedimientos realizados en ámbito de especializada u hospitalario por no existir datos al respecto.
En nuestra opinión los resultados sugieren un coste más bajo que en el ámbito hospitalario, dada la mayor complejidad de este medio, que utiliza entre otras cosas mayor número de profesionales por procedimiento, presumiblemente más tiempo por proceso, y mayores gastos administrativos y de mantenimiento que seguro dispararían aún más los costes.

Además, la práctica de procedimientos de cirugía menor en Atención Primaria supone para los pacientes un beneficio económico añadido, al no tener que desplazarse hasta el hospital, concertando la fecha más adecuada, evitando bajas laborales innecesarias o la rápida resolución del problema (aliviando la preocupación del paciente), aunque todos estos beneficios no han sido medidos económicamente.

Por último, el abanico de procedimientos, la eficacia en el tratamiento, la satisfacción de los usuarios y el alivio de las listas de espera han sido descritos en diversos trabajos y artículos ${ }^{2-4,19,20}$, pero tampoco fueron evaluados económicamente.

Sería interesante la realización de un análisis coste-beneficio pero obviamente precisamos de más estudios, en el ámbito de primaria como de especializa$\mathrm{da}$, que permitan cuantificar monetariamente los beneficios obtenidos por la implantación de programas de CM en AP. Este análisis permitiría de forma más objetiva la potenciación y extensión de programas de $\mathrm{CM}$ en AP.

Por todo lo anterior concluimos que la falta de estudios de costes sobre $\mathrm{CM}$ en atención especializada en nuestra región impide comparar nuestros resultados aunque, en nuestra opinión, los programas de CM en AP muestran un bajo coste. Se precisa utilizar metodologías estandarizadas de evaluación económica para comparar resultados tanto en AP como especializada. Por último, se hace necesario continuar investigando sobre la cuantificación de los beneficios económicos que supone la práctica de cirugía menor en AP.

\section{AGRADECIMIENTOS}

Agradecemos a la gerencia de Atención Primaria $\mathrm{N}^{\circ} 1$ de Murcia la colaboración prestada para la determinación de los costes materiales y de personal. También queremos mostrar nuestro agradecimiento al Dr. Menárguez Puche y a la Unidad Docente de MFyC de Murcia por su inestimable ayuda en la corrección de este trabajo.

\section{CORRESPONDENCIA}

José Cruzado Quevedo

C/ Mistral, 6. Urb. Los Vientos

30500 Molina de Segura. Murcia

Tel.: 968614174

e-mail: jcruzadoq@papps.org 


\section{Bibliografía}

1. Menon NK. Minor surgery in general practice. The Practitioner 1986; 230: 917-20.

2. Alcántara Muñoz P, Menárguez Puche JF, Berenguer Pina JJ, Meseguer Copado MJ, Llorens Minguell A, Hernández Soler J. Cirugía menor en un centro de salud. Experiencia en los seis primeros meses [Panel]. VI Congreso Internacional de Medicina Familiar y Comunitaria. Organizado por la Sociedad Española de Medicina Familiar y Comunitaria. Granada: 13-16 Noviembre, 1996.

3. Arribas Blanco JM, Rodríguez Salceda I, Mena Mateo JM, Martín Martín S, Bru Amantegui S, Villarroel Rodríguez J. Cirugía menor en la consulta del médico de familia. Descripción de un año de experiencia. Aten Primaria 1996; 2: 142-6.

4. Arribas Blanco JM, Gil Sanz ME, Sanz Rodrigo C, Morón Merchante I, Muñoz-Quirós Aliaga S, López Romero A, et al. Efectividad de la cirugía menor dermatológica en la consulta del médico de familia y satisfacción del paciente en relación con la cirugía ambulatoria. Med Clín 1996; 107: $772-5$.

5. Tárraga López PJ, Marín Nieto E, García Olmo D, Celada Rodríguez J, Solera Albero J, Cerdán Oliver M, et al. Impacto económico de la implantación de un programa de cirugía menor en Atención Primaria. Aten Primaria 2001; 5: 335-8.

6. Vaquero Martínez JJ, García Aparicio J, Díaz Gómez J, Blasco Paredes D. Eficiencia de la cirugía menor en atención primaria según el nivel de facturación. Aten Primaria 2002; 30 (2): 86-91.

7. Brown JS. Minor operations in general practice. $\mathrm{Br} \mathrm{J}$ Med 1979; 1: 1609-10.

8. Brown JS. Should general practitioners perform minor surgery? [Editorial]. Br J Hosp Med 1988; 39: 103.

9. Godfrey E, Watkiss M, Schnieden H. Initiation and evaluation of a pilot scheme for minor surgery in general practice. Health Trends 1990; 2: 57-9.

10. Brown JS, Ridsdill Smith R, Cantor T, Chesover D, Yearsley R. General practitioners as providers of minor surgery a success story. Br J Gen Pract 1997; 27: 205-10.
11. Shkrank AB. General practitioners and minor surgery [Comment]. Br J Dermatol 1991; 125: 599-600.

12. Shkrank AB [Letter]. Health Trends 1990; 4: 181.

13. Bowling A. Research methods in health. Investigating health and health services. Buckingham. Philadelphia: Open University Press, 1997.

14. Drummond M F, O'Brien B, Storddart GL, Torrance GW. Methods for the Economic Evaluation of Health Care Programmes. 2. ${ }^{a}$ ed. Oxford: Oxford University Press, 1997.

15. McGuire A, Henderson J, Mooney G. The Economics of Health Care: An Introductory Text. London: Routledge, 1995.

16. Aday LA, Begley Ch E, Lairson DR, Slater CH. Evaluating the Medical Care System: Effectiveness. Efficiency and Equity. Ann Arbor, Michigan: Health Administration Press, 1993.

17. Berenguer E, Costa J. Aplicación y limitaciones de los estudios de evaluación económica en el ámbito sanitario. Jano. Medicina y Humanidades 1999; 56: 61.

18. Menárguez Puche JF, Alcántara Muñoz PA, Cruzado Quevedo J, Llorens Minguell A, Ruiz Puerta MD, Álvarez Orcajada MJ. Cirugía menor en un centro de salud. Inicio de una experiencia [Comunicación]. IV Congreso de Atención Primaria de Salud. Organizado por la Sociedad Murciana de Medicina Familiar y Comunitaria. Lorca (Murcia): 13-15 de Junio; 1996.

19. Luna Rodríguez C, Menárguez Puche JF, Alcántara Muñoz PA, Aroca García MD, Aguilar Martínez J. Experiencias y actitudes en C.M. de los sanitarios de la región de Murcia [Comunicación panel]. VI Congreso Internacional de M.F y C. Organizado por la Sociedad Española de Medicina Familiar y Comunitaria. Granada: 13-16 Noviembre; 1996.

20. Alcántara Muñoz PA, Menárguez Puche JF, Martínez Villalba MJ, Rubio Espín A, Martínez Gonzálvez AB, Albaladejo Serrano AP. Crioterapia en atención primaria: eficaz y segura. [Comunicación panel]. XIX Congreso Nacional de Medicina Familiar y Comunitaria. Organizado por la Sociedad Española de Medicina Familiar y Comunitaria. Tenerife: 8-11 Diciembre; 1999. 